\title{
Die südlichen Trebser Schotter aus dem Oberpliozän Südwest-Mecklenburgs
}

\author{
Werner von Bülow \& Dieter Hans Mai *)
}

\author{
Late Pliocene, early pleistocene, fluvial sediments \\ Trebser Schotter, gravel analysis, heavy minerals, fossil plants, facies, hydrography \\ South-West Mecklenburg
}

\begin{abstract}
Kurzfassung: Die Bohrung Trebs 13/65 auf dem Salzstock Lübtheen (Krs. Hagenow, Südwestmecklenburg) ist bisher der einzige Aufschluß, in dem die Trebser Schotter nachgewiesen sind. Bereits mehrfach erwähnt, wird das Bohrprofil reproduzierbar dokumentiert.
\end{abstract}

In der gröberen Fraktion der $78 \mathrm{~m}$ mächtigen (-14 bis -92 m NN) Trebser Schotter herrschen Quarz-, Kieselschiefer- und Porphyr-Gerölle vor. Die feinere Fraktion führt eine Zirkon-Turmalin-Rutil-Schwermineralgemeinschaft. Aus der den Trebser Schottern eingeschalteten Mudde wurde eine Makroflora isoliert, die mit dem osteuropäischen Kinel' parallelisiert wird.

Die fluviatilen Trebser Schotter werden in das mittlere OberPliozän gestellt. Die Loosener Schotter, die das Tertiär diskordant überlagern, sind jünger. Auf Grund des hohen Kieselschiefer-Anteils wird für die Trebser Schotter eine Herkunft aus Süden bis Südwesten angenommen. Damit ergeben sich neue Aspekte für die Rekonstruktion der faziellpaläohydrographischen Verhältnisse an der Wende NeogenPleistozän im südwestlichen Mecklenburg.

[The southern Trebser gravel from the upper pliocene of Southwest Mecklenburg]

Abstract: The borehole Trebs $13 / 65$ on the saltdome (District Hagenow, Southwest-Mecklenburg) is till now the only exposure, where the Trebser gravel has been proved. As already sometimes mentioned, the profile of a bore will be reproducible established by documentary evidence.

The coarser fraction of the $78 \mathrm{~m}$ thick $(-14$ to $-92 \mathrm{~m} \mathrm{NN})$ Trebser gravel is dominated by quartz-, Kieselschiefer- and porphyr pebbles. The finer fraction contains a zircon-tour-

*) Anschrift der Verfasser: Dr. W. von BüLow, Geologisches Landesamt Mecklenburg-Vorpommern, Industrie-Str. 5, O-2781 Schwerin. Dr. D. H. MAI, Museum für Naturkunde der Humboldt-Universität zu Berlin, Institut für Spezielle Botanik und Botanische Sammlungen, Arboretum, Späth-Str. 80/81, O-1195 Berlin. maline - rutile - heavy mineral association. Out of the mudde which was intercalated in the Trebser gravel a flora could be isolated, which could be connected with the east european Kinel.

The fluviatile Trebser gravel is related to the middle part of the upper pliocene. The Loosner gravels, which superimpose the tertiary strata discordantly, are stratigraphically younger. Because of the high content of Kieselschiefer there has to be postulated for the Trebser gravel a southern or southwestern origin. Therefore there are new aspects for the reconstruction of the conditions of facies and hydrography at the boundary Neogene-Pleistocene in Southwest-Mecklenburg.

\section{Einleitung}

Die Trebser Schotter wurden bereits von LOTSCH (1981) als oberpliozäne Ablagerungen in den Standard der ehemaligen DDR, TGL 25234/08 „Stratigraphische Skala der DDR, Tertiär" aufgenommen. KRUTZSCH (1988) kam aufgrund paläobotanischer Untersuchungen $\mathrm{zu}$ der gleichen stratigraphischen Einstufung. 1991 informierte BÜLOW über deren fazielle und paläohydrographische Ausdeutung. Eine ausführliche und damit reproduzierbare Dokumentation über die Trebser Schichten fehlt jedoch bis heute.

Die Bohrung Trebs 13/65 ist der erste und bisher einzige Aufschluß, aus dem die gleichnamigen Schichten bekannt sind. Sie gehört zum Forschungsprogramm „Neogen Südwestmecklenburg”, dessen Ergebnisse in einem unveröffentlichten Bericht von 1970 dokumentiert sind. Als Trockenbohrung niedergebracht, bot das gewonnene Probenmaterial gute Voraussetzungen für umfangreiche Untersuchungen. MAI bestimmte 1965 die Makroflora. Die Schwermineralanalysen wurden 1966 im ehemaligen Institut für angewandte Mineralogie Dresden vorgenommen. Die Korngrößenbestimmungen stammen aus dem Erd- 
baulabor des VEB Geologische Forschung und Erkundung Nord-Schwerin und die Geröllzählungen führte BüLOW im Jahr 1975 aus.

\section{Geologische Situation}

Die Bohrung Trebs 13/65 liegt im Kreis Hagenow (südwestliches Mecklenburg) ca. $3 \mathrm{~km}$ südöstlich von
Lübtheen in der Gemeinde Trebs (s. Abb. 1). Die Geländeoberfläche um Lübtheen ist eben und besteht aus überdünten Talsanden des Weichsel-Hochglazials.

Unterhalb der Bohrung befindet sich der Top des Salzstockes Lübtheen, dessen Gipshut in Trebs bei $-172 \mathrm{~m} \mathrm{NN}$ erbohrt wurde. Das Diapirstadium des Salzstockes begann im mittleren Miozän und endete

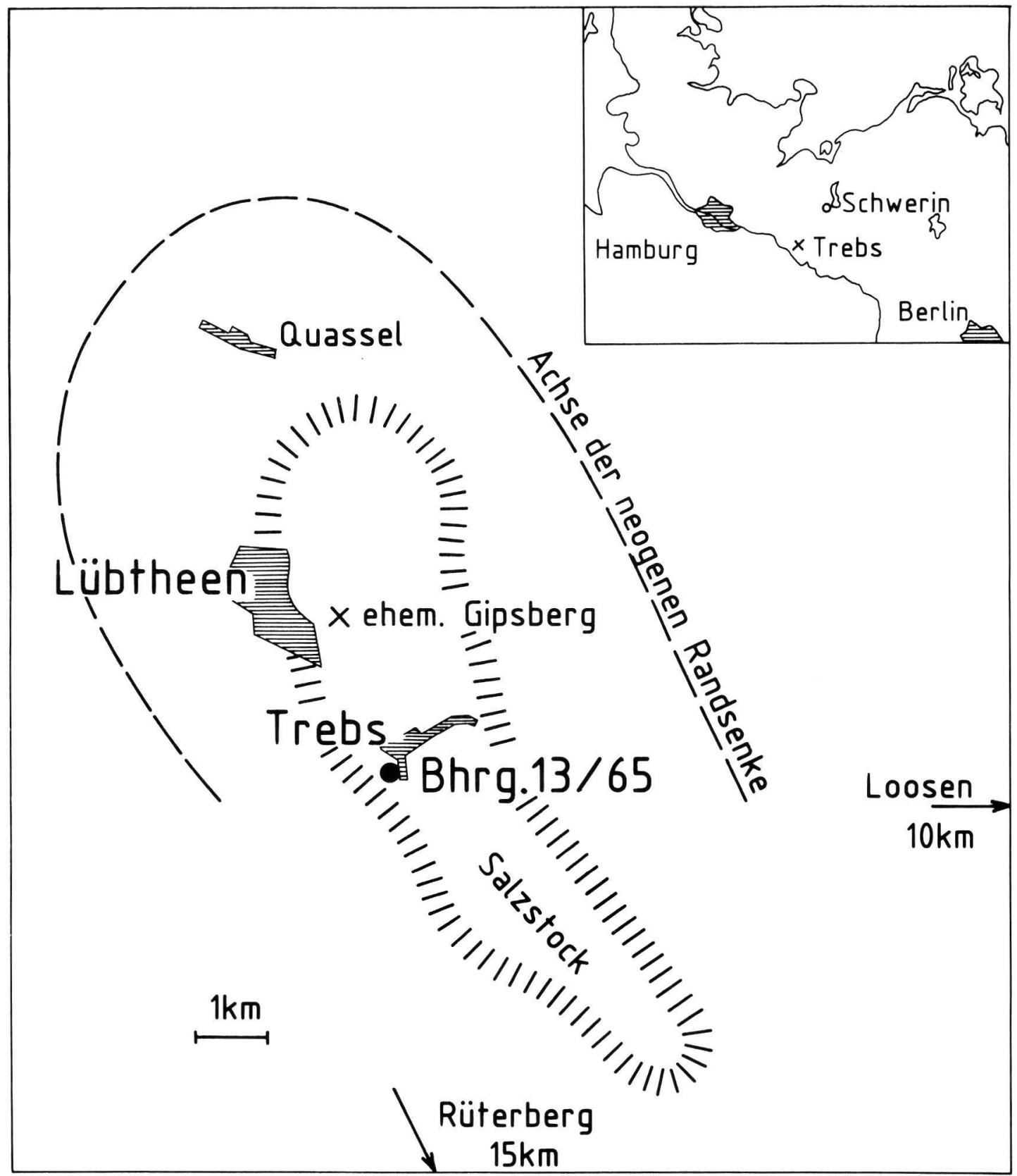

Abb. 1: Lageskizze der Bohrung Trebs 13/65. 
(?) im Holozän; in seiner sekundären Randsenke wurde die Basis des mittelmiozänen Glimmertons unter $-900 \mathrm{~m} \mathrm{NN}$ nachgewiesen. Jüngste Bewegungen im Nordteil des Salzstockes sind durch einen Gipsberg bei Lübtheen belegt, der bereits im vorigen Jahrhundert vollständig abgebaut wurde. Über dem mittleren Teil des Diapirs endeten die Hebungen bereits früher, denn dort sind obermiozäne bis oberpliozäne Ablagerungen erhalten $-z$. B. bei Trebs.

In der weiteren Umgebung stehen an mehreren Stellen oberpliozäne (?) Loosener Schotter an (KRUTZSCH 1988). Sie müssen jünger als die Trebser Schotter sein, denn im Rüterberger Vorkommen (AHRENS et al. 1968) treten an ihrer Basis Muddegerölle auf, die eine etwa gleichalte Früchteflora enthalten wie sie im Trebser Profil nachgewiesen ist:

Tafel / Figur:

Chamaecyparis obtusa SIEB.

et ZuCC.

$\mathrm{I} / 5-8$

Carex vesicaria $\mathrm{L}$.

Menyanthes trifoliata $\mathrm{L}$.

Brasenia bresciana DOROF.

III/9-11

Cladiocarya europaea (DOROF.)

MAI

Carpolithus carpinoides MAI

Pinus brevis LUDW.

Pinus saturni UNG.

I/9-10

I/ $11-12$

Hypericum calcycinoides MAI

Taxodium dubium (STERNB.) HEER

Epipremnites reniculus (LUDW.)

GREGOR et BOGNER

Dulichium arundinaceum (L.)

BRITT.

Caricoidea globosa

(C. et E. M. REID) MAI

Selaginella selaginoides (L.) LINK

Gaylussacia europaea MAI

Andromeda polifolia L.

Chamaedaphne calyculate (L.)

MOENCH

Tubela fusca DoroF.

Glyptostrobus brevisiliquata

(LUDW.) MAI

Carpolithus sp. 4

Nuphar lutea (L.) SM.

Salix sp.

Chenopodium vulvaria $\mathrm{L}$.

Caldesia jacutica DOROF.

Carex pseudocyperus L.

Carex nigra (L.) REICHARD

Punica antiquorum (HEER) MAI

Nymphaea alba L.

Decodon sibiricus DOROF.
II $/ 6-7$

II $/ 24-26$

II $/ 19-21$

III $/ 28-31$

III $/ 1-5$

$\mathrm{I} / 1-2$
Tafel / Figur:

Vitaceen-Ranken

Potamogeton coloratus HORNEM.

Azolla tegeliensis FLORSCHÜTZ

Carex sp. indet.

Lycopus europaeus L.

Empetrum nigrum $\mathrm{L}$.

Eleocharis palustris (L.)

RoEm et SCHULT.

Viburnum sp.

Wichtige Exoten sind Glyptostrobus brevisiliquata, Pinus brevis, Chamaecyparis obtusa, Decodon sibiricus, Cladiocarya europaea, Tubela fusca und Gaylussacia europaea. Als borealmontane Formen sind Andromeda polifolia, Chamaedaphne calyculata, Empetrum nigrum und Selaginella selaginoides anzusehen, die für den Kinel'-Florentyp in Osteuropa sprechen (WELICZKIEWICZ 1982, 1990). Andererseits bestehen aber durch diese und einige andere Arten auch deutliche Beziehungen zu der oberpliozänen Flora des Vildštejn-Komplexes. Nach der Florengliederung von MAI \& WALTHER (1988) ist die Rüterberger Flora nicht ganz sicher in den Florenkomplex Reuver-Berga einzuordnen.

Zwischen beiden Schottertypen befindet sich jedoch eine wesentliche Diskordanz: während sich die Trebser Schotter (Basis $-92 \mathrm{~m} \mathrm{NN}$ ) kontinuierlich aus dem liegenden Mio- und Pliozän entwickeln, überlagern die Loosener Schotter (Basis bei $+40 \mathrm{~m} \mathrm{NN}$ ) diskordant Schichten des tieferen Eozän, des Chatt, des Unter- und Obermiozän.

Vor Ablagerung der Loosener Schichten müssen die halokinetischen (und tektonischen?) Verstellungen im südwestlichen Mecklenburg demnach weitgehend abgeschlossen gewesen sein. Andererseits können die Loosener Schotter als älteste Hochterrasse zu den übertiefen Rinnen des Altpleistozäns angesehen werden, deren Basis nahe Hagenow bei ca. $-400 \mathrm{~m}$ NN nachgewiesen ist (BÜLOW 1967). In ihnen ist mächtiges Pleistozän von Präelster bis Saale abgelagert und erhalten. Außerhalb der Rinnen auf den präpleistozänen Hochflächen kann glazigenes Pleistozän vollständig fehlen. Über den Trebser Schottern sind beispielsweise ausschließlich glazifluviatile Sedimente erbohrt.

Die Trebser Schotter tragen - zusammen mit den Loosener Schottern - dazu bei, die an der Grenze Plio-Pleistozän sich ändernden paläohydrographischen Verhältnisse in Südwestmecklenburg aufzuklären (BÜLOW 1991). Darüber hinaus kann mit ihrer Hilfe die Entwicklung der Salzstrukturen präzisiert werden. 


\section{Profilbeschreibung}

Im Liegenden beginnt das Trebser Profil mit limnischem tonigem Schluff (s. Abb. 2). Er ist vorwiegend schwarzbraun, enthält aber häufig gelbliche, graue, violette oder grünliche Schlieren und Lagen. Sandund Humusgehalt sind gering und Karbonate fehlen. Stellenweise sind Pflanzenreste und Xylite anzutreffen. Die Schichtung - soweit erkennbar - zeigt intensive Verwürgungen, die auf subaquatische Rutschungen zurückgeführt werden könnten.

In einem Übergangsbereich (111 bis $123 \mathrm{~m}$ unter Geländeoberfläche) setzen sich die beschriebenen Pelite fort, doch werden sie durch fein- bis grobkörnige Quarzsandschüttungen unterbrochen, die zu der hangenden fluviatilen Serie überleiten.

Die eigentlichen Trebser Schotter sind $78 \mathrm{~m}$ mächtig (111 bis $33 \mathrm{~m}$ unter Geländeoberfläche). Es handelt sich um kalkfreie feinsandige Mittelsande mit häufigen grobsandigen bis feinkiesigen Partien. In diese fluviatile Folge ist in der Teufe zwischen 55 und $35 \mathrm{~m}$ eine zyklische Kornverfeinerung (mittelsandiger kiesfreier Feinsand) eingeschaltet, die in einem Schluff zwischen 52 und $47 \mathrm{~m}$ unter Geländeoberkante kulminiert. Bei letzterem handelt es sich um eine bröckelige bis geschichtet-aufblätternde dunkelgraubraune kalkfreie Mudde mit klastischen Lagen und zusammengeschwemmten Pflanzenresten.

Im Hangenden der Trebser Schotter folgen bis $13 \mathrm{~m}$ unter Geländeoberkante Mittelsande mit grobsandigkiesigen Einschaltungen, die schon makroskopisch als pleistozäne Schmelzwasserbildungen erkennbar sind. Oberhalb von $13 \mathrm{~m}$ werden sie von Feinsanden mit wechselndem Mittelsandanteil überlagert.

\section{Geröllzählungen}

Wegen der durchweg geringen Korngrößen der Klastika mußte für die Geröllzählungen auf die Fraktion 2,5-20 mm zurückgegriffen werden. Um das Transportverhalten der einzelnen Gerölle einschätzen zu können, wurde die Fraktion 5 bis $20 \mathrm{~mm}$ gesondert ausgehalten und dargestellt (vgl. Abb. 2). Die Zählungen beziehen sich hauptsächlich auf die Trebser Schotter; zur Erfassung der Plio-Pleistozängrenze wurden lediglich 2 Proben aus den Schmelzwassersanden analysiert. Aus dem liegenden Übergangsbereich war kein geeignetes Probenmaterial gewinnbar. Die Bestimmungen der Einzelgerölle erfolgte unter dem Binokular.

Hauptziel der Untersuchungen war es, die generelle Herkunft der Trebser Schotter zu ermitteln. Dafür erschien die Zuordnung der Gerölle in 5 Gruppen ausreichend:
- die Quarze der Feinkiesfraktion sind durchweg gut gerundet, meist klar, aber auch milchig oder unterschiedlich gefärbt. Es treten auch Rest- und Porphyrquarze auf. Die gröberen Gerölle sind weniger gut gerundet und in der Feinsandfraktion herrschen splittrige Bruchstücke vor.

- Sandstein/Quarzit ist eine Sammelgruppe, die wegen ihrer meist geringen Korngrößen nicht weiter differenziert wurde.

- Die Gerölle der Gruppe Porphyr/Feldspat sind fast ausnahmslos hellgrau gebleicht. Etliche von ihnen zeigen Fließstrukturen und enthalten idiomorphe Quarze. Zwischen Porphyren und Feldspäten bestehen vielfältige Übergänge, die eine Zusammenfassung zu einer Gruppe sinnvoll erscheinen ließen.

- Die Kieselschiefer bilden die auffallendste Geröllgruppe. Sie sind meist schwarz, polyedrisch und häufig von Quarzgängen durchzogen. Ihre relative Häufigkeit steigt in den gröberen Fraktionen auf Kosten des Quarzes.

- Unter Sonstige verbergen sich Silizifikate, Kieselgelite, Hornsteine, Pyritaggregate, Opalsandstein, Amethyst, Jaspis, Kieseloolithe u.a. In einigen Proben wurden die massenhaft auftretenden Lokalgerölle wie Diatomeenkohle oder Xylite dieser Gruppe zugeordnet.

Gruppen nordischer Gerölle (frisches Kristallin, Feuersteine, paläozoische Kalke) konnten lediglich in den 2 obersten Pleistozänproben ausgehalten werden. In den Trebser Schottern sind bestenfalls einzelne Gerölle typisch für nordischen Einfluß. Die entscheidenden Gruppen Kieselschiefer und Porphyr/Feldspat beweisen dagegen südliche Herkunft, zumindest der groben Lagen. Herrn BARTHOlOMÄUS, Hannover, der 2 Proben durchsah, gelang es ebenfalls nicht, ,... in dem Trebser Material etwas sicher nordisches zu finden" (briefl. Mitteilung 1991).

Die durchschnittliche Zusammensetzung eines Teils der gezählten Proben wurde bereits früher veröffentlicht (BÜLOW 1991). Hier nun das Mittel aller Zählungen aus den Trebser Schottern (Stück-\%):

$$
2,5-20 \mathrm{~mm} \quad 5-20 \mathrm{~mm}
$$

Quarz

Sandstein/Quarzit

Porphyr/Feldspat

Kieselschiefer

Sonstige

66

41

7

9

14

4

8

7

39

5

Nach der Häufigkeit der 3 wichtigsten Geröllgruppen können die Trebser Schotter als Quarz-KieselschieferPorphyr-Gemeinschaft bezeichnet werden. Aus der 
m U. GOK

Bhrg. Trebs $13 / 65$

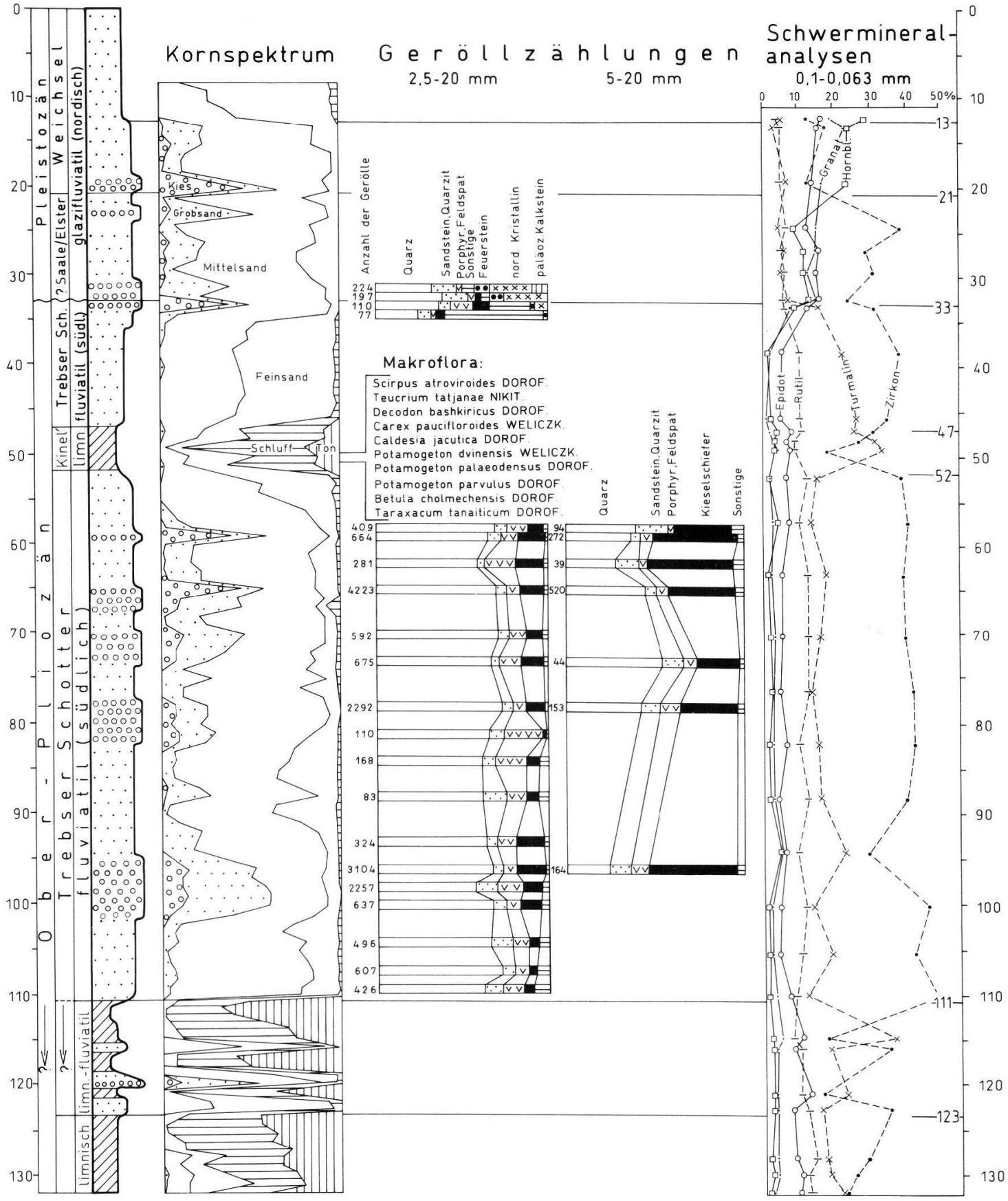

Abb. 2: Dokumentationsprofil und Deutung der Bohrung Trebs 13/65. 
Abnahme des Kieselschiefergehaltes in der feineren Fraktion wird abgeleitet, daß keine transportbedingte relative Anreicherung dieser Gesteine erfolgt ist. Das Herkunftsgebiet der Schotter muß demnach ausgesprochen viel Kieselschiefer geliefert haben; mehr jedenfalls, als bisher aus dem Elbeeinzugsgebiet - aus Südosten - bekannt ist (UNGER 1974, EISSMANN 1975, WOLF 1977, RUSKE 1973). Es ist folglich nicht auszuschließen, daß die Trebser Schotter aus Süden (Westharz) oder gar aus Südwesten (Weser-Einzugsgebiet) geschüttet sein könnten. An der Weser haben PREUSS (1975) und ROHDE (1989) in präglaziären Schottern hohe Kieselschiefergehalte festgestellt. Damit sind erstmalig südliche Einflüsse in den sonst rein nordischen Kaolinsanden des jüngsten Neogen nachgewiesen (BIJLSMA 1981).

\section{Schwermineralanalysen}

Mit Hilfe der Schwermineralanalysen ist das gesteckte Ziel erreicht worden, das Trebser Profil zu untergliedern. Die Verwitterungsauslese nimmt vom Liegenden zum Hangenden sprunghaft $a b$, so daß sich drei unterschiedlich stabile Schwermineralgemeinschaften abzeichnen. Deren Bezeichnung erfolgte in der Reihenfolge der drei am häufigsten auftretenden Mineralgruppen:

1. Zirkon-Turmalin-Rutil-Gemeinschaft: Trebser Schotter mit eingeschalteter Mudde und limnischen Ablagerungen im Liegenden, wobei in den schluffigtonigen Bereichen der Zirkongehalt zugunsten von Turmalin zurückgeht.

2. Zirkon-Epidot-Granat-Gemeinschaft: glazifluviatiles Pleistozän von 21 bis $33 \mathrm{~m}$ unter Geländeoberkante. Die Liegendgrenze entspricht exakt der geröllanalytisch ermittelten Quartärbasis. Da im Hangenden eine instabilere Mineralgemeinschaft folgt, kann das Pleistozän zweigeteilt werden. Der untere Teil wird vorbehaltlich der Saale- oder Elster-Kaltzeit zugeordnet.

3. Hornblende-Granat-Epidot-Gemeinschaft: Weichsel-zeitliche glazifluviatile Sande, die sich aus liegendem kiesig-grobsandigem Mittelsand (Niederterrasse ?; 21 bis $13 \mathrm{~m}$ unter Geländeoberkante) und hangendem Feinsand (Talsand) zusammensetzen.

Für eine Bewertung der Schwermineralführung bezüglich ihrer Herkunft fehlen ausreichende Vergleichsmöglichkeiten. Hohe Zirkon- und Turmalinwerte sowie einzelne Topase, Korunde, Brookite, Monazite und Olivine könnten auf südliche Beeinflussung hindeuten. Die hohen Gehalte an Zirkon und Turmalin treten nach Untersuchungen von Herrn Prof. Henningsen, Hannover, nur noch in den vermutlich gleichalten, ebenfalls südlich beeinflußten
Quasseler Schichten aus der Lübtheener Randsenke auf (briefliche Mitteilung 1991). In den nordischen Kaolinsanden von Sylt herrschen dagegen Epidot, Staurolith, Sillimanit und Disthen vor, während Zirkon, Turmalin und Rutil zurücktreten (DiETHELM 1990).

\section{Paläobotanik}

Aus der Mudde (49,8 - 50,8 m unter Geländeoberkante), die in die Trebser Schotter eingeschaltet ist, wurde eine reiche Früchteflora isoliert und bestimmt (Reihenfolge nach Häufigkeit):

Scirpus atroviroides DOROF.

Tafel / Figur:

Alisma plantago-pliocenica NIKIT.

II $/ 22-23$

Najas lanceolata C. et E. M. REID

I/ $20-23$

I/ $18-19$

Andromeda polifolia L.

III $/ 26-27$

Teucrium tatjanae NIKIT.

III/ $18-20$

Epipremnites reniculus (LUDW.)

GREGOR et BOGNER

II $/ 4-5$

Decodon bashkiricus DOROF.

Hypericum tetrapterum FRIES

Lycopus europaeus L.

III / $14-16$

Typha latifolia L.

Ludwigia palustris (L.) ELLIOTT

Schoenoplectus lacustris (L.)

PaLla

Carex paucifloroides WELICZK.

Carex pseudocyperus L.

Eleogiton (Isolepis) fluitans

(L.) LINK

II / $11-12$

Carex vesicaria $\mathrm{L}$.

Rubus idaeus L.

III/ 13

Caldesia jacutica DOROF.

Nuphar lutea (L.) SM.

Chamaedaphne calyculata (L.)

MOENCH

Catabrosa aquatica (L.) P. B.

Potamogeton dvinensis WELICZK

$P$. palaeodensus Dorof.

III $/ 17$

I/ $15-17$

III $/ 21-22$

II / $13-14$

$P$. parvulus Dorof

Polygonum lapathifolium L.

Carex caespitosa L.

Weigela szaferi ŁAN-S̄RODOŃIOWA

Salvinia rhenana KEMPF

Salix sp.

Najas marina L.

Potentilla pliocenica E. M. REID

Betula cholmechensis DOROF.

$B$. pendula RoTH.

III $/ 6-7$

$\mathrm{III} / 8$

Mentha aquatica L.

Taraxacum tanaiticum DOROF.

III $/ 23$

Salvinia cerebrata NIKIT.

Equisetum sp.
II/ $17-18$

III/ $24-25$ 
Tafel / Figur:

Sagittaria sagittifolia $\mathrm{L}$.

I/ 14

Typha hercynica MAI

Brasenia bresciana DOROF.

Carex binervis SM.

II $/ 16$

C. davalliana SM.

II $/ 15$

Polygonum pliocenicum DOROF.

Potentilla arenaria BORKH.

$\mathrm{III} / 12$

Bemerkenswert ist, daß in der Trebser Wasser- und Sumpfpflanzengesellschaft viele Exoten (z. B. Salvinia cerebrata und rhenana, Epipremnites reniculus, Brasenia bresciana, Alisma plantago-pliocenica, Weigela szaferi, Potentilla pliocenica, Polygonum pliocenicum und Najas Lanceolata) und atlantische Arten (Ludwigia palustris, Carex binervis, Eleogiton fluitans) vorkommen. Andererseits überrascht das gehäufte Auftreten von Arten des osteuropäischen Kinel'-Typs der Russischen Plattform: Scirpus atroviroides, Teucrium tatjanae, Decodon bashkiricus, Carex pauciflorides, Caldesia jacutica, Potamogeton dvinensis, P. palaeodensus, P. parvulus, Betula cholmechensis und Taraxacum tanaiticum (DOROFEEV 1977, WELICZKIEWICZ 1982, 1990). Vergleichbare Taphozönosen sind von Dvoretz/Dnjepr, Anikschtschaj/Litauen, Starodworzy und Siwkovo/Njemen bekannt.

Die Kinel'-Flora wird in den Grenzbereich zwischen Kimmerien und Aktschagyl, bzw. in das „Prätiglian” der westeuropäischen Gliederung gestellt. Nach der ostdeutschen Florenkomplex-Gliederung ist sie am wahrscheinlichsten mit dem Perrier/RippersrodaMaximum gleichzusetzen.

In einem Korrelationsschema, dem eigene Sporomorphen-Untersuchungen zu Grunde liegen, kommt KRUTZSCH (1988) zu einer nahezu gleichen Einstufung. Danach sind synchron mit der Trebser Flora abgelagert worden: Suderode, Willershausen und Görsbach (Harz-Umrandung), Oberzella (Thüringen), Allershausen (Niedersachsen), Reuverton s. str. (Niederlande), Reuver der Quasseler Schichten (SW-Mecklenburg). KRUTZSCH parallelisiert die Trebser Schotter außerdem mit der Ellerhoop-Warmzeit aus dem schleswig-holsteinischen Känozän von MENKE (1975)!

Da die florenführende Mudde eine autochthone Bildung innerhalb der Flußschotter darstellt, wird das oberpliozäne Alter der gesamten Trebser Schotter als gesichert angesehen. Durch diese präzisierte Einstufung und die Geröllzählungen wird die fazielle und paläohydrographische Rekonstruktion am Ende des Neogens in Südwest-Mecklenburg (BÜLOW 1991) untermauert, auch wenn die Flußschotter als isoliertes Vorkommen auf einem Salzstock keine Aussagen über eine mögliche Terrassen-Zugehörigkeit zulassen.

\section{Schriftenverzeichnis}

Ahrens, H., Bülow, W. von, CepeK, A. G., Erd, K., Jähnichen, H., Krutzsch, W., Lotsch, D., Mai, D. H. \& STEINMÜlLeR, A. (1968): Zur Plio/Pleistozän-Grenze in der Deutschen Demokratischen Republik. - Proced. XXIII Internat. Geol. Congress, 10, 65-77, $5 \mathrm{Abb}$; Praha.

BijLSMA, S. (1981): Fluvial Sedimentation from the Fennoscandian Area into the north-west European Basin during the late Cenozoic. - Geologie en Mijnbouw, 60, S. 337-345; Dordrecht.

BÜLOW, W. vON (1967): Zur Quartärbasis in Mecklenburg. - Ber. deutsch. Ges. geol. Wiss., A, Geol. Paläont., 12, H. 3/4, S. 405-413; Berlin.

- (1969): Altpleistozäne Schotter (Loosener Kiese) in Südwestmecklenburg mit nordischen und südlichen $\mathrm{Ge}$ röllen. - Geologie, 18, H. 5, S. 563-589; Berlin.

- (1990): Zur Entstehung der altpleistozänen Rinnen im Bezirk Schwerin. - Z. geol. Wiss., 18, H. 10, S. 939-946; Berlin.

- (1991): Präpleistozäne und Holstein-zeitliche Flußschotter im südwestlichen Mecklenburg. - Z Z geol. Wiss., 19, H. 3, S. 252-260; Berlin.

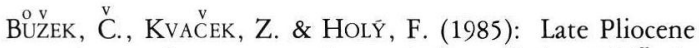
palaeoenvironment and correlation of the Vildstsejn floristic complex within Central Europe. - Rozpr. Ceskoslov. Akad. Vèd, r̀. Matem. a Prírodn., 95 (7): 1-72, 3 Fig., 4 Tab., 20 Taf.; Praha.

Diethelm, R. (1990): Schwermineralzählungen im Kaolinsand von Sylt. - Fossilien von Sylt III, S. 317-324; Hamburg.

DorofeEv, P. I. (1977): Simbuginskaja flora. - In: GoRETZKIJ, G. I. (Hrsg.), Fauna i flora Simbugino (Opornyj razrez akćagyla $i$ apscherona Baschkirii). - $233 \mathrm{~S}$., 24 Abb., 13 u. 56 Taf., Izd. „Nauka”; Moskva.

- (1986): O pliocenovoj flore der. Dvoretz na Dnjpre. In: Takhtadjan, A. L. (Hrsg.), Problemy Paleobotaniki. Sbornik naučnych trudov: 44-71, 7 Fig., 8 Taf., Izd. „Nauka”; Leningrad.

EIssmann, L. (1975): Das Quartär der Leipziger Tieflandsbucht und angrenzender Gebiete um Saale und Elbe. Schriftenr. geol. Wiss., 2, 263 S.; Berlin.

Jakubovskaja, T. V. (1984): Očrerk Neogena i rannego Antropogena Poneman'ja (po paleokarpologičeskim issledovanijam). - 160 S., 20 Fig., 30 Tab., 10 Taf., Izd. „Nauka i Technika”; Minsk.

KRUTZSCH, W. (1988): Kritische Bemerkungen zur Palynologie und zur klimastratigraphischen Gliederung des Pliozäns bis tieferen Altpleistozäns in Süd-, Südwest-, Nordwest- und pro parte Mitteleuropa sowie die Lage der Pliozän/Pleistozän-Grenze in diesem Gebiet. Quartärpaläontologie, 7, S. 7-51; Berlin.

(LoTSCH, D. 1981): TGL 25234/08 Geologie, Stratigraphie, Stratigraphische Skala der DDR, Tertiär. ZGI Berlin. 
MAI, D. H. (1967): Die Florenzonen, der Florenwechsel und die Vorstellungen über den Klimaablauf im Jungtertiär der Deutschen Demokratischen Republik. Abh. zentr. geol. Inst., H. 10: 55-81, 4 Abb., 1 Tab., 2 Taf.; Berlin.

— \& Walther, H. (1988): Die pliozänen Floren von Thüringen, Deutsche Demokratische Republik. - Quartärpaläontologie, 7, 55-297, 101 Abb., 2 Tab., 41 Taf.; Berlin.

MENKE, B. (1975): Vegetationsgeschichte und Florenstratigraphie Nordwestdeutschlands im Pliozän und Frühquartär. Mit einem Beitrag zur Biostratigraphie des Weichselfrühglazials. - Geol. Jb., A 26, S. 3-151; Hannover.

Preuss, H. (1975): Gliederung und Zusammensetzung der Weserterrassen-Körper bei Bodenfelde. - Mitt. geol. Inst. techn. Univ. Hannover, 12, S. 5-51; Hannover.

RoHDE, P. (1989): Elf pleistozäne Sand-Kies-Terrassen der Weser: Erläuterung eines Gliederungsschemas für das obere Weser-Tal. - Eiszeitalter u. Gegenwart, 39, S. 42-56; Hannover.
RUSKE, R. (1973): Stand der Erforschung des Quartärs in den Bezirken Halle und Magdeburg. - Z. geol. Wiss., 1, H. 9, S. 1065-1086; Berlin.

UNGER, K.-P. (1974): Quartär in: Geologie von Thüringen, hrsg. W. Hoppe; G. SeIdel: - S. 742-781; Gotha/ Leipzig.

WeLICZKIEWICZ, F. Ju. (1982): Osnovnye etapy razvitija pliocenovoj flory Evropejskoj casty SSSR. - In: WELICZKIEWICZ, F. JU. (Hrsg.); Paleokarpologiceskie issledovanija Kajnozoja: 104-118, Izd. „Nauka i Technika”; Minsk.

- (1990): The Late-pliocene floras of Byelorussia. Proceed. Symposium "Paleofloristic and Paleoclimatic changes in the Cretaceous and Tertiary": 273-276; Prague.

Wolf, L. (1977): Präglaziale Flußläufe zwischen Dresden und Riesa. - Z. geol. Wiss., 5, H. 6, S. 791-803; Berlin.

Manuskript eingegangen am 28.10. 1991 


\section{Tafeln I-III}



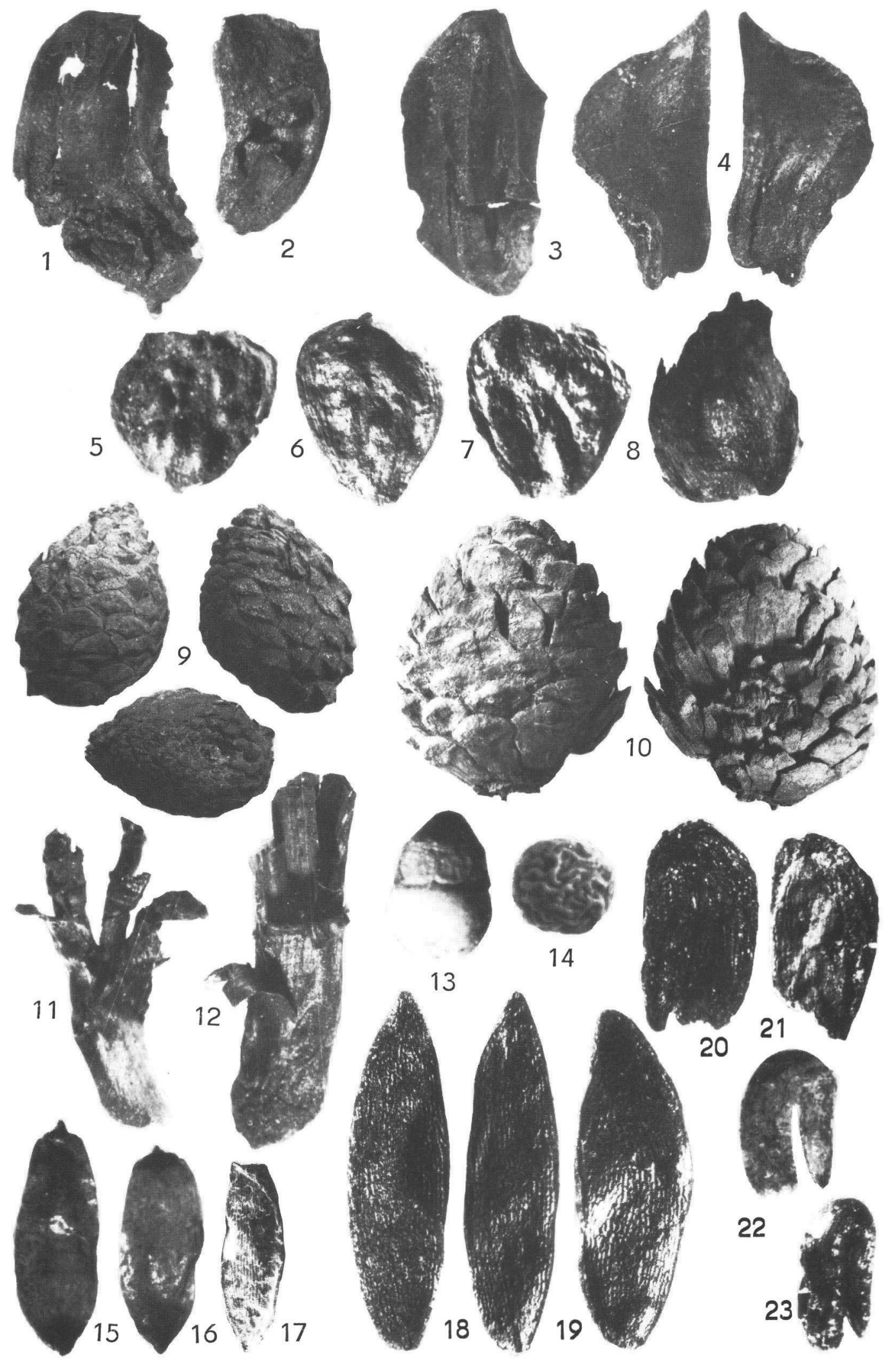


\section{Tafel I}

1-2 - Glyptostrobus brevisiliquata (Ludw.) MAI, Samen, x 10;3-4-Taxodium dubium (STBG.) HeER, Samen, x 6,5;5-8 - Chamaecyparis obtusa SIEB. et ZuCC., Samen, x 10; 9-10-Pinus brevis Ludw., Zapfen von verschiedenen Seiten, 1/1; 11-12 - Pinus saturni UNG., Nadelbasen mit Schuppen, $\mathrm{x}$ 10; 13 - Azolla tegeliensis FlORsCHÜTZ, Megasporangium, x 40; 14 - Salvinia cerebrata Nikitin, Megaspore, x 40;15-17 - Typha latifolia L., Samen, x 40;18-19-Najas lanceolata C. et E. M. REID, Samen, x 20; 20-23 - Alisma plantago-pliocenica NikiT., Früchtchen (Fig. 20-21) und Samen (Fig. 22-23), x 20. 

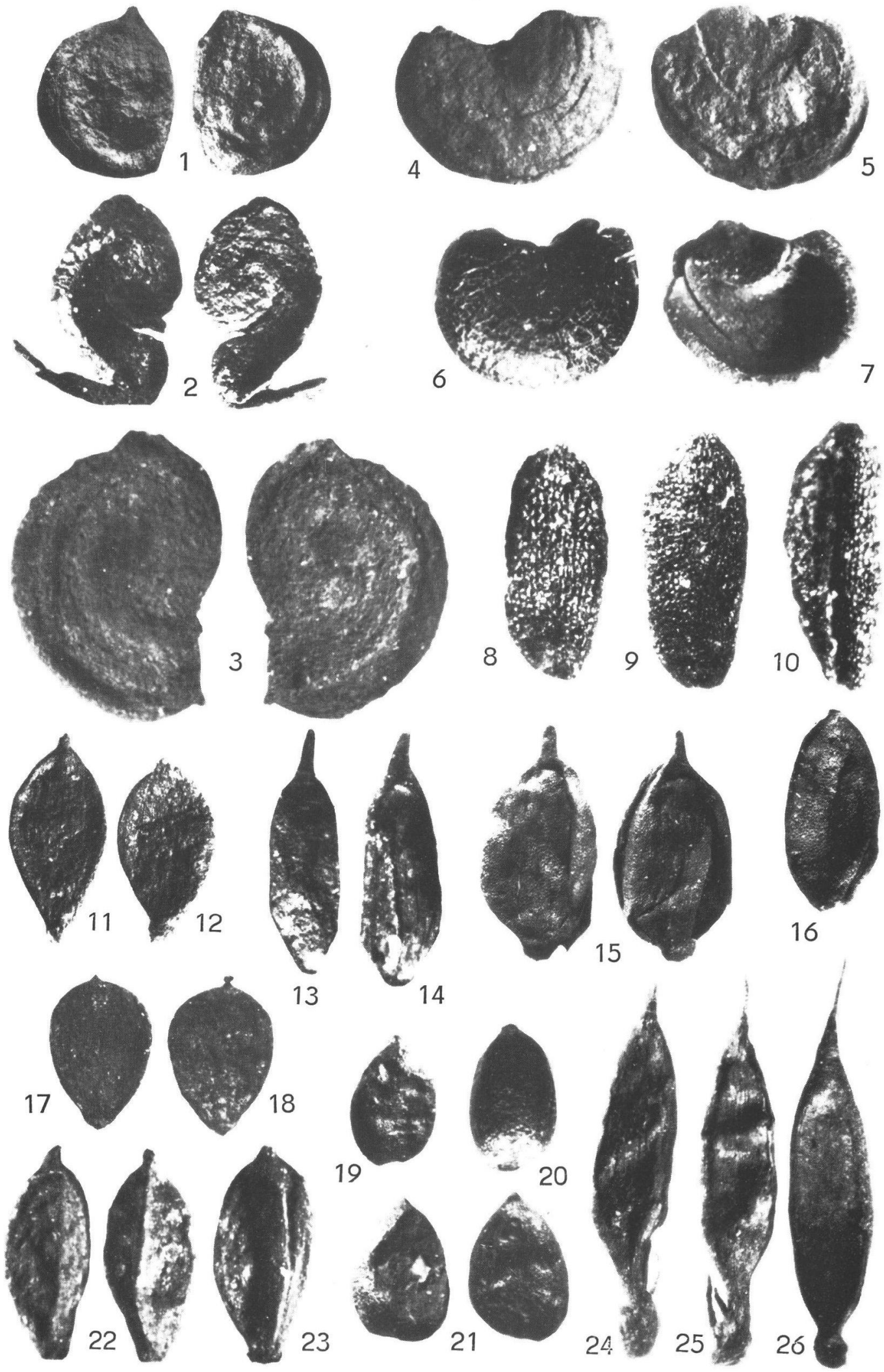


\section{Tafel II}

1 - Potamogeton parvulus Dorof., Steinkern, x 20;2 - Potamogeton palaeodensus Dorof., Steinkern, x 20;3 - Potamogeton dvinensis WeLICZK., Steinkern, x 20;4-5-Epipremnites reniculus Gregor et BOGNER, Samen, x 12; 6-7-dgl., x 12; 8-10-Catabrosa aquatica (L.) P. B., Karyopsen ohne Spelzen, x 40;11-12 - Eleogiton fluitans L., Nüßchen, x 20;13-14 - Carex paucifloroides WeuczK., Nüßchen, x 15; 15 - Carex davalliana SM., Nüßchen von zwei Seiten, x 15;16-Carex binervis SM., Nüßchen, x 15; 17-18 - Carex caespitosa L., Nüßchen, x 15; 19-21 - Caricoidea globosa (E. M. REID) MAI, Früchte, x 15; 22-23 - Scirpus atroviroides DOROF., Früchtchen, x 40; 24-26 - Dulichium arundinaceum (L.) BRITT., Früchtchen, x 20 . 


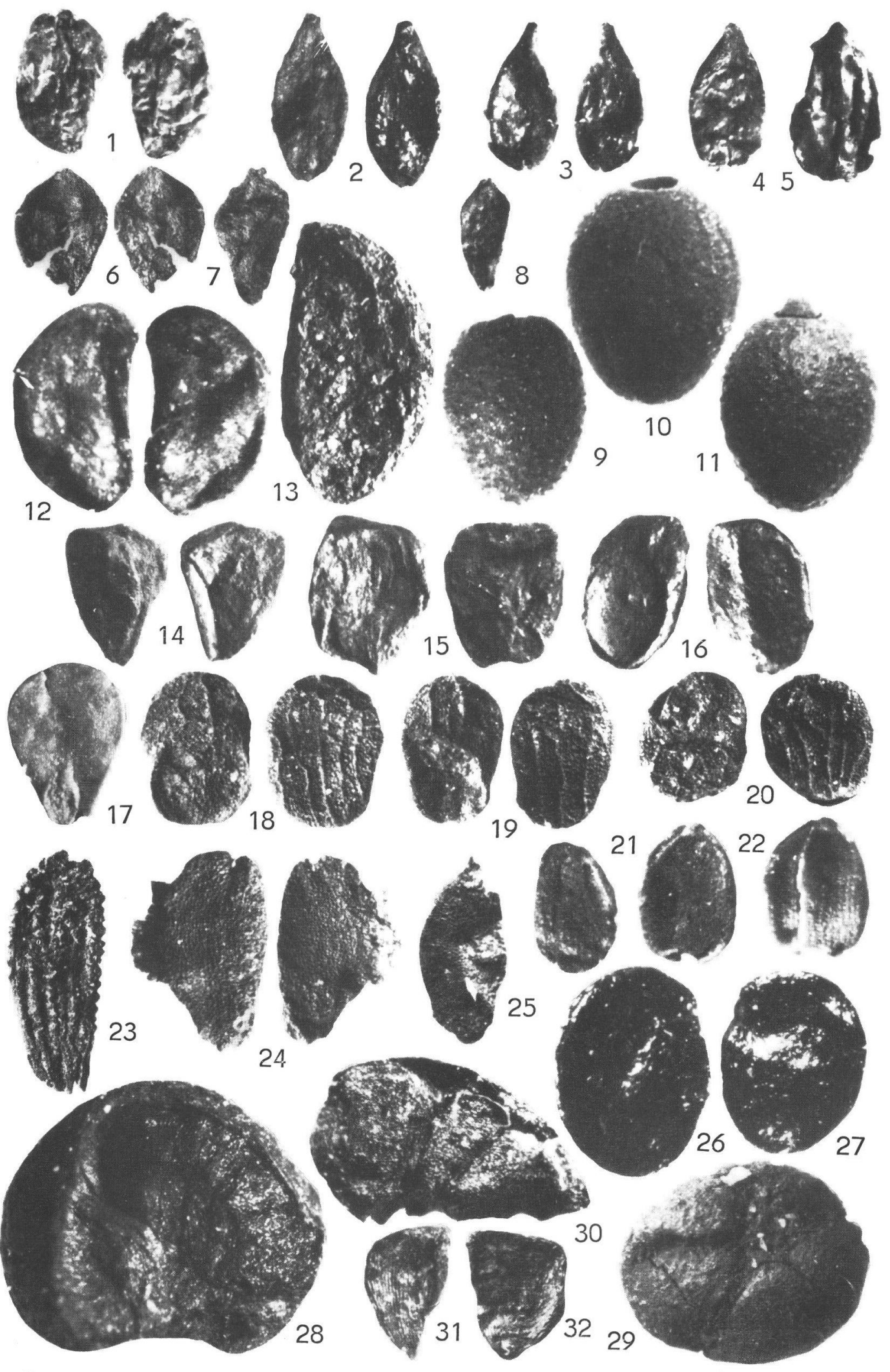




\section{Tafel III}

1-5 - Tubela fusca Dorof., Früchtchen, x 12;6-7-Betula cholmechensis Dorof., Früchtchen ohne Flügelreste, x 12; 8-Betula pendula ROTH., Früchtchen, x 12; 9-11-Brasenia bresciana DoRof., Samen, x 15; 12 - Potentilla cf. arenaria BorkH., Nüßchen, x 30;13-Rubus idaeus L., Steinkern, x 20;14-16 Decodon bashkiricus Dorof., Samen jeweils von beiden Seiten, x 20; 17 - Lycopus europaeus L., Nüßchen ohne Korksaum, x 25; 18-20 - Teucrium tatjanae NIKIT., Nüßchen von dorsal und ventral, x 25; 21-22 Ludwigia palustris (L.) ElLIOTT, Samen, x 40; 23 - Taraxacum tanaiticum Dorof., Achäne, x 20; 24-25Weigela szaferi ŁAN.-S̄RODONIOWA, Samen mit Flügelresten, x 20; 26-27-Andromeda polifolia L., Samen, x 30; 28-32-Chamaedaphne calyculata (L.) MOENCH, Teile von Kapselfrüchten und isolierte Samen, x 30. 